\title{
Observation and Analysis of an Electrically Active Layer at the Core-Shell Interface of a GaN Nanowire by Advanced Electron Microscopy
}

\author{
S. Yazdi ${ }^{1}$, T. Kasama ${ }^{2}$, R. Ciechonski ${ }^{3}$, J. B. Wagner ${ }^{2}$, R. E. Dunin-Borkowski ${ }^{4}$ and E. Ringe ${ }^{1}$ \\ ${ }^{1 .}$ Department of Materials Science \& Nano Engineering (MSNE), Rice University, Houston, Texas, \\ United States \\ 2. Center for Electron Nanoscopy, Technical University of Denmark, Lyngby, Denmark \\ 3. GLO-AB, Ideon Science Park, Lund, Sweden \\ 4. Ernst Ruska-Centre for Microscopy and Spectroscopy with Electrons and Peter Grünberg Institute, \\ Forschungszentrum Jülich, Jülich, Germany
}

Research into GaN-based materials over the last two decades has led to the development of light emitting diodes (LEDs) that span the entire visible spectrum. Their efficiency is expected to be improved significantly by the use of core-shell GaN nanowires as templates for the growth of LEDs. When compared with their planar counterparts, core-shell nanowire LEDs have a larger surface area to volume ratio and therefore a higher light extraction efficiency. Common structural defects in GaN thinfilms that are detrimental for lighting applications, such as threading dislocations, can also be suppressed in nanowires. More importantly, the active regions of LEDs, i.e., quantum wells (QWs), can be grown on exposed non-polar $\{1 \overline{1} 00\} \mathrm{m}$-planes in $c$-axis-oriented nanowires. The presence of inherent spontaneous and piezoelectric fields in wurtzite crystal structures results in the separation and reduction in overlap of the electron and hole wavefunctions in QWs grown along the $c$-axis and therefore degrades the radiative recombination efficiency. It is therefore particularly desirable to grow LEDs on nonpolar $m$-planes. However, here we report the presence of an unexpected electrostatic potential barrier across $m$-planes in core-shell $\mathrm{GaN}$ nanowires. The origin of this potential barrier is investigated using different advanced transmission electron microscopy (TEM) techniques.

The nanowires that are studied here were grown on pre-patterned GaN templates using catalyst-free metal organic chemical vapour deposition in two steps: the core was nucleated and grown vertically to a desirable length and the shell was then grown laterally. Cross-sectional TEM specimens were prepared both along the $a$-planes and perpendicular to the $c$-axis using focused ion beam (FIB) milling [1]. Offaxis electron holography of the interface between the nanowire core and the laterally grown shell on the $m$-planes revealed a potential barrier running along the nanowire length at the interface (see Fig. 1). Electron beam induced current (EBIC) signals were detected by other groups at the same position in similar nanowires, confirming the electrical activity of this potential barrier [2]. High-resolution TEM (HRTEM) shows several 0.5-nm-thick layers within the 25-40-nm potential barrier layer (Fig. 2a). Changes in polarity between the nanowire core and shell was excluded as a possible origin of the observed potential barrier by HRTEM and high-resolution bright-field scanning TEM (BF STEM) (see Fig. $2 b$ and c). The nanowires are identified as N-polar in both the core and the shell. Local strain and/or unintentional doping are likely to be responsible for the electrostatic potential barrier between the core and the shell. 


\section{References:}

[1] S. Yazdi et al. Journal of Physics: Conference Series, 471 (2013), p. 012041.

[2] M. Tchernycheva et al. Nanoscale, 7 (2015), p. 11692.
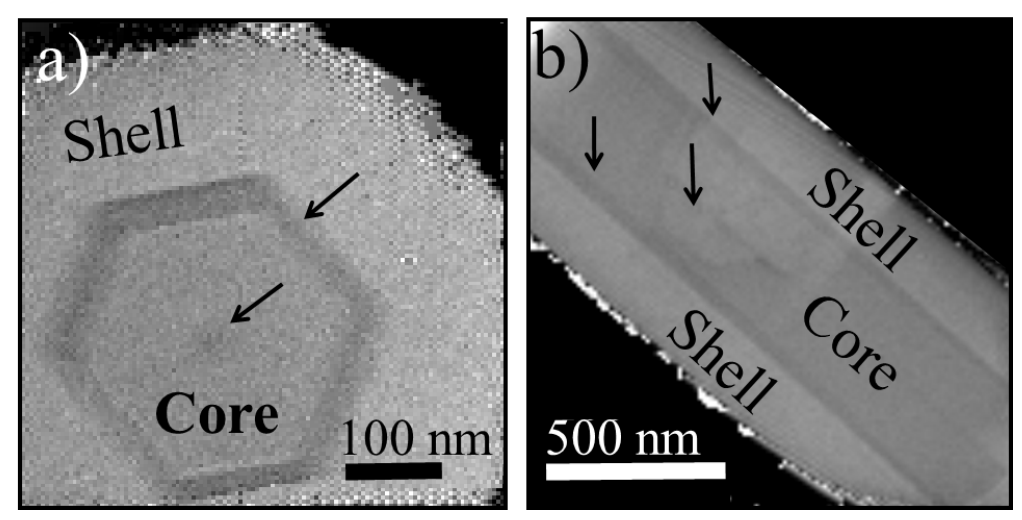

Figure 1. Electrostatic potential maps measured using electron holography from $\mathrm{GaN}$ nanowires prepared using FIB milling a) perpendicular to the $c$-axis and b) parallel to the $a$-planes. The maps reveal a layer between the core and the shell and a region at the center of the core with a lower potential. Arrows point to unexpected potentials. Electron holograms were acquired at $120 \mathrm{kV}$ in an FEI Titan 80-300 TEM equipped with a biprism.

Figure 2. Atomic-resolution images of the interface between the core and the shell of a GaN nanowire. a) HRTEM image showing 0.5-nm-thick layers (marked with yellow stripes) within the potential barrier layer shown in Fig. 1. The red arrow marks the growth direction. b) Enlargement of the region marked by a box in panel a. c) BF STEM image of a different region in the nanowire shell. Panels $\mathrm{b}$ and $\mathrm{c}$ are consistent with N-polar GaN. The images were acquired at $300 \mathrm{kV}$ in a double-Cscorrected FEI Titan Themis 60-300 S/TEM.
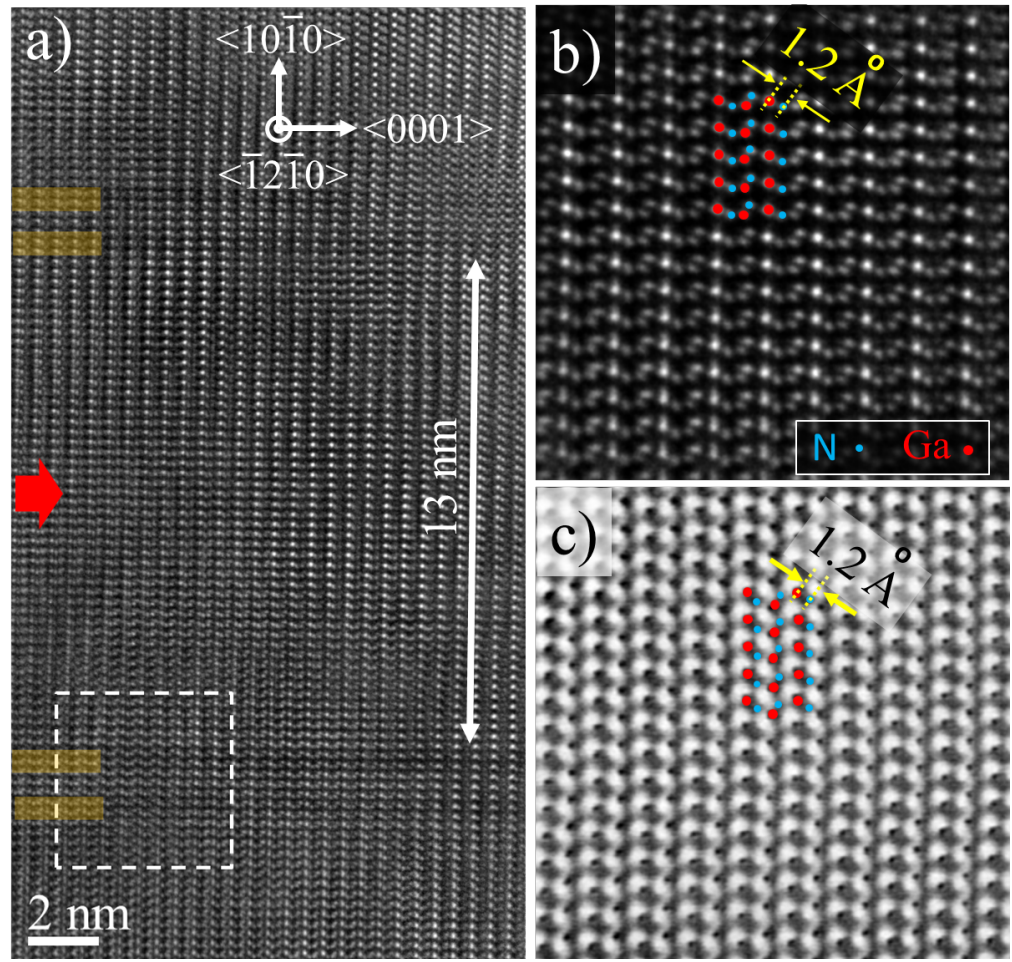